\title{
Research on Green Prevention and Control Technology of Cucumber Diseases and Insect Pests in Solar Greenhouse based on Knowledge Base
}

\author{
Zhiheng Song ${ }^{1}$, Hang Zhang ${ }^{1}$, Naixiang Li $^{1}$ \\ ${ }^{1}$ College of Computer and Information Engineering, Tianjin Agricultural University, Tianjin, 300384
}

Keywords: Green Prevention, Control Technology, Solar Greenhouse

\begin{abstract}
The main conditions for the occurrence of diseases and insect pests in greenhouse vegetables are temperature, humidity, bacterial sources, insect sources, disease resistance and cultivation measures. The ecological control of greenhouse vegetables is controlled by ecological factors such as agriculture, physics and biology. The amount of chemical pesticides eliminates the pollution residue, so that people can eat safer and control the pests and diseases.
\end{abstract}

\section{Introduction}

Cucumber is a popular vegetable and the most common vegetable cultivated in the solar greenhouse. According to the survey of the greenhouse base, the output per $667 \mathrm{~m} 2$ is generally around $6500 \mathrm{~kg}$. According to the wholesale price of 2.5 yuan $/ \mathrm{kg}$, the economic benefit is 16,250 yuan, minus the productive input of 5000. Yuan, with a net benefit of 11,250 yuan. However, due to the high temperature and humidity environment of the solar greenhouse, the pests and diseases occur seriously, which affects the economic income of the majority of vegetable farmers. Therefore, it is necessary for the vegetable farmers to master the high-yield cultivation techniques and pest control techniques for scientifically planting solar greenhouse cucumbers.

\section{Main prevention targets and strategies}

The main diseases of cucumber include blight, stagnation, downy mildew, gray mold, anthracnose, blight, target spot, powdery mildew, black spot, blight, Pseudomonas fuliginea, bacteria Sexual plaque, viral disease (ToMV, CMV, TLCV and AMV) and southern root knot nematode disease.

The main pests of cucumber include aphids, whitefly, whitefly, thrips, Liriomyza sativae, and sassafras.

In accordance with the plant protection policy of prevention and comprehensive prevention and control, we adhere to the principle of agricultural control, physical control, biological control, supplemented by chemical control. Adopt green prevention and control combined with supporting cultivation techniques, and prevent and control strategies combining emergency prevention and early prevention.

Implement a strict rotation system. The solar greenhouse used for cucumber production should be rotated by two crops including green manure. It is advisable to cultivate solanaceae vegetables with more organic fertilizers and less fertilizer, as well as green onions, garlic, and onions that can alleviate cucumber diseases. In areas where conditions permit, water early rotation or summer irrigation should be implemented. It advocates the application of ETS microbial preparations, high-efficiency, low-toxic soil remediation preparations, chitosan, chitooligosaccharides and microbial compounding techniques for soil remediation and improvement. For the main pest and disease control objects in the cultivation season and local restrictions on cucumber production in the solar greenhouse, high-resistance and multi-resistant cucumber varieties were selected, such as cucumber varieties that were resistant to TMV, Fusarium wilt and black star disease. 3.1.4 Cultivate strong seedlings... Use seedling tray aseptic seedling method to raise seedlings to ensure the cultivation of disease-free and age-appropriate seedlings that meet the quality; for plots with serious soil-borne diseases, first consider breeding resistant rootstocks for grafting and seedling; before 
planting Spray 1 time oligosaccharide plant immune inducer, S-excitation, and low temperature refining to improve immunity and stress resistance. The air humidity in the solar greenhouse is controlled by covering the mulch or the inter-row grass; the temperature in the greenhouse is adjusted by the air release and the auxiliary heating to meet the suitable temperature of the cucumber in different growth periods, and the temperature is too high or too low. According to the relationship between temperature and condensation in the solar greenhouse, the temperature of the cover is lowered to control condensation. The use of nitrogen fertilizer should be reduced to reduce the occurrence of pests and diseases; the nutrients such as calcium fertilizer and silicon fertilizer should be applied to enhance the resistance of cucumbers to pests and diseases. The biogas slurry filtered with fine gauze is diluted 150 ...200 times to spray cucumber leaves, which can control cucumber blight and anthracnose, and the control effect can reach more than $90 \%$. At the same time, it has certain curative effects on whitefly, aphids and mites.

Hanging 30 to 40 yellow or blue boards per $667 . . . \mathrm{m} 2$ to trap pests such as aphids, whiteflies and thrips; covering the silver-gray membrane to repel aphids. Lepidoptera pests such as Spodoptera litura are trapped by vibrating insecticidal lamps, black light lamps, and trapping solar insecticidal lamps. The vents and import and export are provided with 40 to 50 mesh insect nets to prevent the entry of mites and white mites. In the solar greenhouse during the leisure period from late June to early August, full use of solar energy can be used for disinfection. Specific operation method: After clearing the upper crop, evenly apply fresh chicken manure $1 . . .000$ to...2...000...kg per $667 \ldots m 2$, crop straw $2 . . .500 \sim 3 \ldots 000 \ldots \mathrm{kg}$, deep soil layer $25 \sim \ldots 40 \ldots \mathrm{cm}$, leveling and simmering; watering makes the relative humidity of the soil reach $85 \%$ to $100 \%$; the mulch covers, and the shed film is closed 25 to $30 \ldots$ d. Introduced and released parasitic natural enemies such as the bee larvae to control pests such as whiteflies. When the adult whitefly was found, the bee was started. Generally, 10,000 heads per $667 . . . \mathrm{m} 2$; if there are more than 5 adult larvae in a single plant, they can be smoked in the whole shed with aerosols to reduce the number of insects, and then put the bees after 7...d. (2) Introduce and release predatory mites such as courgettes and cockroaches to control pests such as thrips. At the seedling stage, each plant released 5 to 10 heads of fresh courgettes, and the result period was 20 to 30 heads per plant. (3) The use of parasitic bacteria such as Beauveria bassiana to control pests such as thrips. Use 20 billion (cfu) / g of Beauveria bassiana powder to dilute to 100 million (cfu) / g of bacteria solution sprayed with water 1:1, the bacterial liquid should be used with the mixture. (4) Use of doxorubicin to control thrips. Spray with $25 . . . \mathrm{g} / \mathrm{kg}$ doxorubicin suspension 800 to $1 . . .000$ times. (5) Use plant source preparations to control a variety of pests such as aphids, spotted fly, and whitefly. Available in $0.3 \%$ azadirachtin EC...800 to $1 . . .200$ times solution, $0.5 \%$ cucurbitacin solution 400 to 600 times solution, or $1 \%$ matrine AS...600 times solution, or $0.65 \%$ anisein AS...400 500 times liquid spray. (6) Use of Paecilomyces lilacinus to control root knot nematode disease. At the time of planting (applied to the roots of the seedlings), 10 billion (cfu)/g of Paecilomyces lilacinus WP...0.5 to 1...kg per 667...m2. (7) Using Bacillus sp. to control various soil-borne diseases such as blight, bacterial wilt and root rot. 1 billion (cfu)/g of Bacillus polymyxa WP...1...000 to 2...000 times, or 1 billion (cfu)/g of Bacillus subtilis WP...1...000 to 2...000 times, or 20 Billion (cfu) / g Bacillus cereus WP ... $200 \sim 300$ times liquid irrigation root, each plant not less than 250 ... g liquid. (8) Use antibiotics to prevent and treat a variety of diseases. 2\% Wuyinmycin AS...250 300 times liquid spray can be used to control gray mold, etc.; 90\% neomycin can be used WP...2...000 3...000 times solution, or 3\% medium oxytetracycline WP...1... $000 \sim 1 . . .200$ times liquid spray to prevent bacterial angular leaf spot; can be used to control gray mold and powdery mildew by 2\% spring seromycin AS...600 800 times liquid spray; 2\% nymidinemycin AS...200 250 times Liquid spray to prevent viral diseases. (9) The use of oligosaccharides and polysaccharide preparations to control a variety of fungal diseases. It can be used to control gray mold and anthracnose with $2 \%$ amino oligosaccharide AS...1...000 times liquid spray; it can be sprayed with $0.4 \%$ oligosaccharide AS...300 500 times to prevent sclerotinia, anthracnose and powdery mildew. (10) The use of oligosaccharides and polysaccharide preparations to control viral diseases. $2 \%$ amino oligosaccharide AS...1...000 times solution, or $0.5 \%$ mushroom proteoglycan AS...250 to 300 times solution, or $0.5 \%$ lentinan AS...500 to 600 times liquid leaf 
spray.

\section{Chemical control}

According to the main types of pests and diseases, select the appropriate fungicides, pesticides, etc., and mix them reasonably. When a single pest occurs in the field, targeted control is carried out. When the field pests and diseases reach the prevention and control indicators, organize emergency control. The use of chemical control shall comply with the requirements for the safe use of pesticides (GB4285) and the guidelines for the rational use of pesticides (GB/T8321) (all parts). It is preferred to use the dust method and the smoking method, pay attention to the rotation of medicines, rationally mix and use, and strictly control the safety interval of pesticides. The concentration of the drug is strictly in accordance with the recommended dosage of the pesticide packaging instructions. Tripping disease... In the early stage of the disease, 38\% of the creams of azoxystrobin AS...800 times, or $72 \%$ of the cream urea・manganese zinc WP...1...000 times solution, or $50 \%$ enoylmorpholine WG...1...500 Double solution, or 72.2\% chlorpyrifos AS...600 times liquid spray or root irrigation control. Blight disease... In the early stage of the disease, $15 \%$ carbendazim AS...500 times solution, or 50\% thiram WP...500 times solution, or $50 \%$ carbendazim WP...600 ..800 times solution or root irrigation can be used. Downy mildew... In the early stage of the disease, 69\% Anke Manganese Zinc WP...800 times solution, or 72\% cream urea cyanide manganese zinc WP...600-800 times solution, or 69\% enoylmorpho-manganese zinc WP...800 times Liquid, or $58 \%$ metalaxyl manganese zinc WP...800 $1 . . .000$ times liquid, or $70 \%$ nail cream.......aluminum copper WP...800 times solution, or 60\% pyraclostrobin WG...1...000 $1 . .500$ times liquid spray control. Gray mold disease... In the early stage of the disease, $50 \%$ azoxystrobin WP...3...000 times solution, or 40\% pyrimethanil WP...800 to $1 . . .200$ times, or 50\% isocytoin WP...800 to $1 . . .200$ times Liquid spray control. When using hormone scented flowers, $0.1 \%$ of $50 \%$ procymidone WP can be added to the liquid, or $10 \%$ of procymidone can be used for 260 to $300 \ldots$ g per $667 . . . \mathrm{m} 2$ to close the greenhouse and fumigation control. Anthrax... In the early stage of the disease, 25\% prochloraz EC...1...000 to...1...500 times solution, or 75\% chlorothalonil WP...800 times solution, or 10\% difenoconazole WG...1...500 2...000 times liquid spray control. Bacterial blight... In the early stage of the disease, 40\% flusilazole EC...8...000 times solution, or $30 \%$ metformin WP...600 times solution, or 38\% acesulfame WP...800 times solution spray Prevention and treatment. Target spot disease... In the early stage of the disease, 25\% azoxystrobin SC...1...500 times solution, or 40\% methylpyrazine SC...500 times solution, or 25\% prochloraz EC...1...500 times solution, 40 \% Fluorosilazole EC...8...000 times liquid spray control. Powdery mildew... In the early stage of the disease, $25 \%$ acetaminophen AS...800 ...1...000 times solution, or $10 \%$ phenyl ether methyl carbazole WG...1...500 2...000 times solution, or $75 \%$ chlorothalonil WP...500 to 600 times solution, or 50\% thiophanate-methyl WP...800 to $1 . . .000$ times solution, or 56\% azoxystrobin chlorothalonil WP...600 times solution spray control. Black spot disease... In the early stage of the disease, use 10\% difenoconazole WG...2...000 times solution, or $60 \%$ azole ether.......Dyson WG...1...500 times solution, or $40 \%$ flusilazole EC... 8...000 times solution, or $43 \%$ tebuconazole SC...3...000 times solution, or 50\% carbendazim WP... 600 times solution, or 25\% pyraclostrobin EC...3...000 times liquid spray control. Fusarium wilt... In the early stage of the disease, $43 \%$ tebuconazole SC...3...000 times solution, or $25 \%$ pyraclostrobin EC...3...000 times solution, or 10\% difenoconazole WG...1...500 times available Liquid spray control. It can also be treated with 50\% carbendazim WP...500 times solution, or $70 \%$ thiophanate-methyl WP...1...000 times solution. Pseudomonas sp. root rot... In the early stage of the disease, 50\% carbendazim WP...500 to 800 times solution, or 14\% lycopene copper AS...300 times solution, or 50\% thiram double WP...500 to 800 times solution may be used. Rooting, 250...g per plant. Bacterial angular plaque... In the early stage of the disease, $78 \%$ nitro humic acid copper WP...600 times solution, or 47\% spring ray • Wang copper WP...600 800 times liquid spray control. It can also be used for 50\% tiger gum copper WP...400 times solution, or $77 \%$ copper hydroxide WP...500 times solution for root irrigation. Viral disease... In the early stage of the 
disease, 20\% morpholinium hydrochloride can be used... •...copper WP...300 400 times solution, or $5 \%$ bacteria clear AS...150 250 times solution, or potassium permanganate $1 . . .000$ times solution $1.8 \%$ sodium nitrophenolate $6 . . .000$ times mixed solution spray control. Root knot nematode... $10 \%$ thiazophos GR...4 to $5 . . . \mathrm{kg}$ before planting, or about 2.5...kg for hole application; $2 \%$ avermectin EC...1...200 1 after planting... 500 times liquid, or 50\% phoxim EC...500 600 times liquid root control. Aphids, whiteflies... Available 10\% imidacloprid WP... 2...800 times solution, or 25\% thiamethoxam WG...2...500 to 3...000 times solution, or 40\% acetamiprid WG...1...000 to 2...000 Double solution, or 40\% thiazinone WP...1...000 times liquid spray control. In the early stage of spawning to the early stage of larval hatching, it can be sprayed with $50 \%$ chlorfenapyr WP...2...500 3...500 times solution or 10\% imidacloprid WP...1...000 times solution. Hummer... 20\% compound Liuyangmycin EC...1...000 times solution, or 2\% imidacloprid WP...500 800 times liquid spray control, 25\% thiamethoxam WG...2...500 4...000 times Rooting. Liriomyza sativae... In the early stage of spawning to the early stage of larval hatching, $50 \%$ chlortetracycline WP...2...500 to 3...500 times solution, or 10\% imidacloprid WP...1...000 times solution, or 1\% Avi Salt SC...1...500 times solution, or 1.8\% avermectin EC...3...000 times liquid spray control.

\section{Conclusion}

The Green Light Prevention and Treatment Technology (DB61/T 395-2007) is applicable to the green control of cucumber diseases and insect pests in the solar greenhouse in Shaanxi Province. The prevention and control target is more than $95 \%$ for pest control and more than $85 \%$ for disease control.

\section{Acknowledgements}

Fund Project: Tianjin Agricultural Science and Technology Achievements Transformation and Promotion Project, Integrated Application of Forest Information Disease Early Warning, Diagnosis and Prevention and Control Core Information Technology, Project No. 201704070

\section{References}

[1] Liu Haizhi. Cultivation Techniques of Zucchini in Solar Greenhouse[J]. Modern Agriculture, 2012, (5): 8-9.

[2] Tian Guanglong. Cultivation Techniques of French Zucchini in Solar Greenhouse [J]. Jilin Vegetables, 2011, (2): 8-9.

[3] Zhao Yan, Han Mingzhi. Chemical control technology for cucumber powdery mildew[J]. Henan Agriculture, 2012, (11): 29.

[4] Wang Zhaojun. Comprehensive Prevention and Control of Cucumber Downy Mildew in Winter Greenhouses [J]. Modern Rural Science and Technology, 2009, (05): 29. 